\title{
Why genetic information processing could have a quantum basis
}

\author{
APOORVA PATEL \\ Centre for Theoretical Studies and Supercomputer Education and Research Centre, \\ Indian Institute of Science, Bangalore 560 012, India \\ (Fax, 91-80-3600106; Email, adpatel@cts.iisc.ernet.in)
}

\begin{abstract}
Living organisms are not just random collections of organic molecules. There is continuous information processing going on in the apparent bouncing around of molecules of life. Optimization criteria in this information processing can be searched for using the laws of physics. Quantum dynamics can explain why living organisms have 4 nucleotide bases and 20 amino acids, as optimal solutions of the molecular assembly process. Experiments should be able to tell whether evolution indeed took advantage of quantum dynamics or not.
\end{abstract}

\section{Information}

What is life? About fifty years ago, Erwin Schrödinger attempted to answer this question on the basis of known laws of physics (Schrödinger 1944). His insight has since then inspired many researchers to investigate the molecular basis of a living organism. Chemical bonds explain how atoms bind together to form various molecules. It is possible to take the common elements $\mathrm{H}, \mathrm{C}, \mathrm{N}, \mathrm{O}$, stir them together with some heat and electric sparks, and obtain molecules of life such as water, methane, ammonia, sugars, amino acids, nucleotide bases, and so on. These molecules exist even in the interstellar clouds. It is also not difficult to arrange these molecules in an orderly manner as in a crystal, or jumble them up in a random ensemble as in a gas. But living organisms are neither ordered crystals nor random mixtures of their building blocks. The building blocks of a living organism are linked together in a precise fashion to make functional parts. These links between building blocks are often indirect and not physical; they describe an order amongst the building blocks. Information is the abstract mathematical concept that quantifies the notion of this order amongst the building blocks, and it was this concept that was emphasized by Schrödinger in his seminal work.

It is easiest to quantify information using the framework of communication. When a message is conveyed by one person to another, the measure of the information contained in the message is the increase in the knowledge of the second person upon receiving the message from the first. The larger the number of possibilities for a message, more is the amount of uncertainty removed upon its receipt, and so more is the information contained in it. The simplest message would be just a yes or no, distinguishing amongst only two possibilities. Claude Shannon thus defined the information contained in a message as its entropy; it directly measures the number of possibilities for the message. A repetitious message wastes resources repeating what is already conveyed before. So the information contained in a message is increased by removing correlations amongst its parts; as messages become more efficient, they appear more and more random.

Information thus lives in randomness, but it is not randomness. What distinguishes it from randomness is the sense of purpose, i.e. the message has a meaningful interpretation for the receiver. Of course, that requires a common language which both the sender and the receiver understand. Often a prior agreement fixes the language, but then the agreement itself would have been made by an earlier message. Going back all the way, one can ultimately connect the interpretation of a message to the physical properties of the objects that carry the message. The most primitive messages have to be of this type, and then higher level sophisticated structures can be constructed using them. Trying to figure out what the best language would be in a given situation is too vast an area

Keywords. Amino acid; computation; database search; DNA; enzyme; information; nucleotide base; protein; quantum coherence; quantum mechanics; superposition; unitary evolution 
of investigation; here I concentrate only on information in messages with a fixed language.

It is a characteristic of living organisms to acquire information, interpret it and pass it on, often using it and refining it along the way. This information can be in various forms. It can be genetic information passed on from the parent to the offspring, sensory information conveyed by the sense organ to the brain, linguistic information communicated by one being to another, or numerical data entered in a computer for later use. Living organisms are thermodynamically open non-equilibrium systems. They absorb free energy, and use it to create order within and throw disorder out. The ultimate source of this free energy has to be an interaction that is not in equilibrium. In our world this interaction is gravity; gravity is always attractive and so cannot be in equilibrium - the lowest energy state is a black hole (see for instance, Davies 1998).

Computer science is the mathematical framework for processing information. A computer takes certain information, in the form of an input, and by suitable manipulations converts it into an output. The manipulations are defined by mathematical algorithms and implemented by physical devices. Obviously the types of manipulations that can be carried out are limited by the types of physical devices available. Efficient computers are those that reliably accomplish their tasks using the least amount of resources. Considering the living organisms to be specialized supercomputers, we can study how efficient they are in implementing their tasks.

\section{Optimization}

Darwinian evolution, i.e. survival of the fittest, describes the adaptations of living organisms to their environment. These adaptations have occurred by trial and error explorations, and not as a direct optimal solution to a mathematical problem. Nowadays we understand them in the language of genetics. Genes contain the essential information (i.e. the programme) of life; they tell the rest of the living cell what to do in what circumstances. The circumstances are provided by the environment, while the genes determine the responses. Faithful replication of the genes passes on the information from one generation to the next. Once in a while, chance mutations alter the responses of the genes. If the change is beneficial the organism improves its chances of survival, and if the change is detrimental the organism fades away. It is important to note that mutations are local fluctuations, and cannot bring about large changes in one go. The new organism is always similar to the old one, and not a completely different one. In principle, large changes can be built up from local ones over a long time. On the other hand, small changes cannot get one out of a local optimum. This latter feature is responsible for the wide variety of life we observe, and even though evolution has progressed over a long period, we cannot be sure that it has always discovered the optimal adaptations. Globally optimal features can be only those which are widespread in organisms living under different conditions. One such instance is the language of the genetic information; it has remained unchanged from ancient bacteria to modern human beings. It is worth exploring to what extent it is an optimal adaptation to the available resources and the physical laws, using the principles of computer science. [I find this approach much more appealing than the frozen accident hypothesis (Crick 1968). The pioneering contribution along this lines is: von Neumann 1958.]

Optimization of information processing is essentially driven by two guidelines: (i) minimization of physical resources (time as well as space), and (ii) minimization of errors. These guidelines often impose conflicting demands, but we have learnt how to tackle them in the process of building powerful computers, and it is instructive to analyse that in some detail.

The first step in optimal representation of a message is to break it up in small segments. This is called digitization. Instead of handling a single variable covering a large range, it is much easier to handle several variables each spanning a smaller range. Information of the whole message is maintained by putting together as many as necessary of the smaller range variables, while the instruction set required to manipulate each variable is substantially simplified. This simplification means that only a limited number of processes have to be physically implemented, and only a limited types of physical variables have to be handled, leading to high speed computation*. Furthermore, choosing the smaller range variables to be discrete, and not continuous, it is possible to correct small errors. Continuous variables can drift, and it is not possible to figure out the extent of the drift. Discrete variables based on continuous physical properties can drift too, but they can be reset to the nearest discrete variable, eliminating the error whenever the drift is small**. These advantages of a simple instruction set and error correction are so overwhelming that it has become customary to describe a

\footnotetext{
*We learnt mathematical tables in primary school to carry out addition and multiplication using the decimal system. In binary system used by our computers, these tables are replaced by only two operations, XOR and AND.

**For example, voltages and currents in electrical circuits are continuous variables, but the transistors in digital computer circuits are used only in their discrete saturated states. Small voltage fluctuations are eliminated by resetting the transistors to their saturated states. Only when the voltage fluctuation is large, the state of the transistor flips and there is an error in the calculation.
} 
message containing information as an aperiodic chain of building blocks. Our systems of writing numbers and sentences have such a structure. Genetic information has also incorporated this optimization step: DNA and RNA chains use an alphabet of 4 nucleotide bases, while polypeptide chains use an alphabet of 20 amino acids.

The second optimization step is the packing of the information in a message. Repetitive structures or correlations amongst different parts of a message reduce the capacity of the message to convey information - part of the variables are wasted in repeating what is already conveyed. Elimination of correlations reduces the length of a message; the information content of a fixed length message is maximized when all the correlations are eliminated and each of the variables is made as random as possible. On the other hand, processing errors in an efficiently packed message destroys information. If correlations exist amongst different parts of a message, they can be exploited to eliminate local disturbances and to reconstruct the correct message. Both these features are used in our computers: files are compressed without losing information for efficient storage, and parity checks are routinely performed to detect and reconstruct spoilt data. How efficiently the information should be packed, and how many correlations should be kept, is a trade-off that depends on the error rate of the particular information processing system*. Detailed analyses of DNA sequences have found little correlation amongst the letters of its alphabet in the coding regions, although correlations do exist in the noncoding regions (see for example, Arneodo et al 1995; Nandy 1996). We have to marvel at the fact that evolution has achieved the close to maximum entropy structure of coding the genetic information.

Selection of the number of letters in the alphabet is the third optimization step. It clearly depends on the task to be accomplished and the choices available as building blocks. There must be at least two building blocks for a linear chain to carry information; a periodic crystal of a single building block carries little information. While choosing a large number of building blocks reduces the overall length of the message, it also makes distinguishing them from each other more difficult. The practical criterion for fast error-free information processing is therefore to choose as many letters in the alphabet as can be quickly distinguished from each other. Different physical reasons are involved in the selection of building blocks of different information processing systems: the decimal system

\footnotetext{
*We use one form of language to communicate to adults, and another form to talk to babies. On comparing them, we immediately notice that the "baby language" is full of repetitive syllables - an insurance against high communication loss.
}

arose from our learning to count with our fingers, the number of syllables in our languages are determined by the number of distinct sounds our vocal chords can make, binary code is used in computers and nervous systems because off/on states can be quickly decided with electrical signals. Are there any such physical underpinnings for the number of letters chosen for DNA and protein chains? The task involved in genetic information processing is ASSEMBLY. The desired components already exist (they are floating around in a random ensemble); they are picked up one by one and linked together in the required order. Whether a particular component is the desired one or not is decided by base-pairing, and it is a simple yes/no query - either the base-pairing takes place or it does not. The optimization criterion for this task is now clear: find the number of items that can be reliably distinguished from each other given a fixed number of yes/no queries. This is a mathematically well-defined problem, to be solved using the laws of physics available at the molecular scale.

Many more than 4 nucleotide bases and many more than 20 amino acids can be synthesized by chemical reactions. Indeed many of them exist in the cellular environment. But DNA and RNA always contain 4 nucleotide bases and polypeptide chains always contain 20 amino acids. A number of attempts have been made to understand these mysterious numbers, since the structure of DNA was unraveled. Since DNA replication required complementary base-pairing, it was reasonable to expect the number of nucleotide bases in DNA to be even. Beyond that there was no understanding of why the number of nucleotide bases should be 4 , except that 2 is the smallest possible value and 4 is the next one. Far more elaborate schemes were constructed to explain why polypeptide chains should contain 20 amino acids. They were based on stereochemical properties of the molecules involved, various permutations of the nucleotide bases and combinatorics (Hayes 1998 has a recent summary of these efforts). All these schemes fell apart with the discovery of the non-overlapping triplet code.

\section{Two languages}

Before trying to figure out the optimal number of letters for the DNA and protein alphabets, let us first understand why genetic information processing requires two distinct languages - one with the nucleotide bases as the building blocks and another with the amino acids as the building blocks. We often translate one language into another by replacing one set of building blocks with another. When the languages are versatile enough this translation can be carried out without any loss in the information content; a code specifies which set of building blocks are translated 
into which ones. A particular set of building blocks is selected not by the abstract information content that has to be conveyed, but by its suitability for the physical tasks to be carried out during processing of the information. Our computers compute using electrical signals but store the results on the disk using magnetic signals; the former realization is suitable for quick processing while the latter is suitable for long term storage. Proteins and DNA participate in similar tasks. Proteins are actively involved in many biochemical processes going on in the cell, and suffer much wear and tear as a result. The double helical structure of DNA, with the nucleotide bases hidden inside, carefully protects the information until it is required. Also, DNA replication is much less error-prone than protein synthesis.

This is only part of the story. Translation between DNA and protein languages is more complicated than just swapping one set of building blocks for another. When magnetic signals are converted into electric signals in a computer, the building blocks change but the language does not; both forms represent the same sequence of zeroes and ones. In the case of DNA and proteins, not only the building blocks change, but the language undergoes a change too. The translation would have been easier if only the building blocks changed as per physical requirements, but not the language. Then what necessitated a change in the language? The reason again has to do with the task associated with the information. For example, the textual information typed on the keyboard is stored in a computer in binary format using the ascii code. The textual format is easier for humans to read and speak, while the binary format is easier for computers to manipulate. The job assigned to DNA is faithful replication and transcription, which is easily accomplished in the form of one-dimensional chains of building blocks. The job assigned to proteins is participation in biochemical reactions, where size and shape of the protein play a critical role. The building blocks of proteins - the amino acids have to therefore know how to fold one-dimensional chains into three-dimensional structures. The number of building blocks required to encode one-dimensional chains and three-dimensional structures is certainly different (Patel 2001b). It is this drastic change in physical realization of the information that has driven the living organisms to develop two distinct languages, and the complex machinery that translates one into the other.

There is yet another distinction between the languages of DNA and proteins. The language of DNA is a high level one, analogous to the compact languages we use to write our computer programmes. It is symbolic and abstract, and it requires interpretation before the information contained in it can be used. The language of proteins is a low level one, even more direct than the machine codes which run computers. There is no other agency to interpret the information; the building blocks themselves carry the instructions of what is to be done in terms of their physical properties. The sequence of amino acids "knows" how to fold itself, which in turn decides which biochemical reaction the protein will participate in.

The concepts of information processing and optimization have been in the background of all attempts to understand the features of the genetic code, but the actual code that was discovered did not show any obvious relations to them. (Boolean logic with yes/no queries can only produce 2 and its powers as the possible number of building blocks.) Since then biologists have considered, by and large, the genetic code as a frozen accident of history: it arose somehow and became such a vital part of life that any change in it would be highly deleterious (Crick 1968). The situation changed again, with the realization that quantum logic can be used for information processing as well, and its optimization features are different than those of classical Boolean logic. So we can go back and look again at the same problem, incorporating all that we have learnt in the framework of computer science over the years. The next section outlines how quantum logic explains the mysterious numbers of the genetic code as solutions to an optimization problem (Patel 2000a, b).

\section{Quantum logic}

Quantum mechanics describes our understanding of how atoms are built from their constituents and how they interact with each other. Its dynamical equations are defined in terms of amplitudes (or wavefunctions), and the classical observation probabilities are obtained by squaring the amplitudes. It is important to note that these dynamical equations are precise, the probabilistic interpretation arises only when we convert the dynamical amplitudes into classical observables. The amplitudes are a set of complex numbers, normalized to the total probability being one, and they evolve in time by unitary transformations. Complex amplitudes and their unitary evolution follow a totally different dynamics compared to real probabilities and their Markovian evolution. This difference lies at the basis of why quantum algorithms and their optimization criteria are distinct from their Boolean counterparts. It also makes the quantum logic is superior to the classical one - the same task is accomplished using less resources (time and space).

The ASSEMBLY process is a variation of the SEARCH process, where the desired object is picked up from an ensemble based on certain property checks. In case of genetic information processing, the ensemble is a random one and the property checks are implemented by molecular bonds involved in base-pairing. The dynamics of molecular bond formation is no doubt quantum, and so 
a quantum search algorithm is a possibility for genetic information processing.

The optimal quantum search algorithm was found by Lov Grover (1996), and it relates the number of objects, $N$, that can be distinguished by a number of yes/no queries, $Q$, according to

$$
(2 Q+1) \sin ^{-1}(1 / \sqrt{N})=\pi / 2 .
$$

This algorithm does not use the full power of quantum logic; concepts of superposition and interference familiar from the study of classical waves are sufficient to describe it. The algorithm starts with a uniform superposition of all possible states, corresponding to equal probability for every building block to get selected. Then it applies two reflection operations alternately: (i) change the sign of the amplitude of the desired state by the yes/no query, and (ii) reflect all amplitudes about their average value. The algorithm stops after $Q$ of these alternating reflections to yield the desired state with a high probability.

The solutions of eq. (1) for small values of $Q$ have special significance for the number of building blocks involved in genetic information processing (details of the genetic code can be found in Watson et al 1987; Lewin 2000):

$$
\begin{aligned}
& Q=1 \Rightarrow N=4, \\
& Q=2 \Rightarrow N=10.5, \\
& Q=3 \Rightarrow N=20.2 .
\end{aligned}
$$

(i) A single base-pairing distinguishes between 4 possibilities in DNA replication and mRNA transcription. This is an exact solution of eq. (1), so chances of error are minimized.

(ii) Bilingual tRNA synthetases ensure the matching between the amino acid at one corner of the tRNA molecule and the anticodon triplet at another. These synthetases belong to two distinct classes of 10 each, distinguished by the structure of their active sites and by how they attach amino acids to the tRNA molecules. The lack of any relationship between the two classes have led to proposals that they evolved independently. It is quite plausible that early forms of life existed with proteins that were made up of just 10 amino acids, belonging to one class or the other and coded by two nucleotide bases. The wobble rules and similar codons in the genetic code for amino acids with similar properties would then be relics of the merger of the two distinct classes during evolution (Patel 2001b). $N=10$ is not an exact solution for $Q=2$, which means that the quantum algorithm will not always find the desired object. There exists a small probability, about 1 part in 1000, that the quantum algorithm will select a wrong object.

(iii) Three base-pairings between tRNA and mRNA transfer the information from the nucleotide base chain to the amino acid chain. The non-overlapping triplet genetic code carries 21 signals ( 20 for the amino acids plus a STOP) in this process. $N=21$ is not an exact solution for $Q=3$; the quantum algorithm then has an intrinsic error probability of about 1 part in 1000 .

\section{DNA structure}

Having discovered that the optimal quantum search algorithm can explain the number of building blocks involved in genetic information processing, the next exercise is to look for the physical implementation of the steps of the algorithm. During DNA replication, the intact strand of DNA acts as a template on which the growing strand is assembled. At each step, the base on the intact strand decides which one of the four possible bases in the environment can pair with it. This is exactly the yes/no query used in the search algorithm. Based on the known features of this process, I have proposed the following scenario (Patel 2000a):

(i) The molecular bonds involved in base-pairing are hydrogen bonds. They can be explained only using the language of quantum mechanics, and correspond to quantum tunnelling of a proton $\left(\mathrm{H}^{+}\right)$between two attractive energy minima. When the nucleotide bases come together with random orientations, their pairing takes place in a two-step process. The formation of the first bond still leaves enough freedom for nucleotide bases to rotate and orient in various ways in the three-dimensional space. The second step fully locks the nucleotide bases in their bound structure. Such a two-step base-pairing process has the correct quantum dynamics to flip the sign of the amplitude of the desired state.

(ii) Over a long time, the quantum amplitudes relax towards an equilibrium state. The base-pairing takes place on a very short time scale, and acts as a sudden disturbance. The amplitudes then again try to relax back to the equilibrium state, just like a damped pendulum which is suddenly kicked. The opposite end of the oscillation of the amplitudes about the equilibrium state corresponds to the reflection about average operation. If the quantum algorithm is stopped there by extracting the binding energy, the desired base-pairing is achieved.

(iii) The job of creating the uniform superposition of all states, and then maintaining the coherence of the quantum dynamics is assigned to the enzymes. Enzymes play a crucial catalytic role in the replication process; the process simply does not occur in the absence of enzymes by chance molecular collisions. Disturbances from the environment, called decoherence, are extremely fast and generally destroy quantum features of macroscopic systems in no time. Enzymes do provide shielded environments at the molecular scale, and modern techniques of molecular 
biology can be used to check whether they can offset the effects of decoherence in the case of DNA replication or not (Patel 2001a).

\section{Future}

I have analysed the molecular assembly process from the view-point of information theory and optimization. The best algorithm for accomplishing this task is based on quantum dynamics. The optimal number of building blocks predicted by the algorithm agree remarkably well with the number of building blocks involved in genetic information processing - replication of DNA and synthesis of proteins. The molecular structure and dynamics of base-pairing also has the features necessary to implement the quantum algorithm. The question that remains is: does genetic information processing really use the quantum algorithm, or is the existence of both software and hardware features an accident?

This genetic information processing takes place at the atomic scale, where quantum mechanics is the framework for understanding the physical processes. It is reasonable to expect that if there was something to be gained from quantum algorithms, life would have taken advantage of that at this physical scale. Of course, the structure of DNA came into existence billions of years ago, and it could be that what was relevant when life arose is not relevant now; the observed features could be just left-overs from a bygone era. Even though we cannot recreate the conditions in which life originated, we can experimentally test whether at present quantum dynamics plays a role in genetic information processing or not (Patel 2001a).

The biggest obstacle to quantum dynamics is decoherence, and it is worthwhile to investigate whether living organisms have conquered it in at least some of the fundamental processes of life or not. Experiments should be able to check the quantum scenario for genetic information processing, and then we would know the adequacy of our current understanding of molecular biology.

Comparing genetic information processing to our modern digital computers, we can observe that DNA plays the role of memory, mRNA plays the role of registers, enzymes play the role of instructions, while ribosomes and other complicated structures in the cell carry out the tasks of the CPU. It is the CPU and the instructions that make it work, that form the heart of the computer. Memory is the simplest, and quite likely the last to be developed, component of the computer. Using the language of information theory, therefore, I have looked at only the simplest of the processes. There are deeper questions to be addressed, and more complicated processes to be analysed. For example, what kind of optimization selected particular physical objects as the building blocks? What kind of primitive machinery could have led to the present genetic code? What is the relevance of the degeneracy of the genetic code for amino acids? What is the dynamics behind the catalytic role played by the enzymes? All that is for the future. I can only say that the possible involvement of quantum dynamics in genetic information processing has provided a novel way of analysing these processes.

\section{Acknowledgements}

I am grateful to $\mathrm{S}$ Mahadevan, $\mathrm{V}$ Nanjundiah and $\mathrm{H}$ Sharat Chandra for their helpful suggestions.

\section{Appendix}

Here I describe the meanings of some of the technical words used in this article.

Superposition: For linear dynamical systems, a linear combination of possible solutions is also a solution to the same dynamics. For convenience, a suitable basis is chosen, and a general solution is parametrized by its components along the basis directions. In wave mechanics and nonrelativistic quantum dynamics, these components are complex numbers and are called amplitudes. Superposition corresponds to addition of the amplitudes when combining several solutions. Complex amplitudes can add constructively as well as destructively, leading to interference patterns. Observation probability of a particular solution is given by the absolute square of the corresponding amplitude.

Unitary evolution: A linear dynamical system evolves by a linear transformation of its components, often denoted as multiplication of a vector by a matrix. Conservation of total observation probability implies that the squared-norm of the vector is always unity, and the most general transformation is a rotation. When the components of the vector are complex numbers, the most general evolution matrix is a unitary one.

Markovian evolution: Classical probability theory can also describe linear dynamics, with individual probabilities assigned to every component. Each component probability has to be a real number between zero and one, and the sum of all components is always unity. The elements of the evolution matrix are real numbers between zero and one, such that elements in every column of the matrix add up to one. Such an evolution of probabilities is called Markovian evolution.

Decoherence: Although quantum dynamics provides a perfect description of processes occurring at the atomic scale, we hardly observe any quantum effects at the macroscopic scale. Decoherence is the explanation of how 
interaction of a quantum system with its environment can reduce its quantum behaviour to classical one. Collisions and scatterings carry away complex phases of the quantum system into the surrounding environment, where they are irretrievably lost. A quantum system without its complex phases cannot be described in terms of superposition of amplitudes; it has to be described in terms of "averaged" statistical probabilities. Decoherence can be reduced by insulating the quantum system from its environment, but that becomes highly difficult as the system size increases. In general, decoherence is extremely fast even for microscopic systems - so fast that it has not been directly observed.

\section{References}

Arneodo A, Bacry E, Graves P V and Muzy J F 1995 Characterizing long range correlations in DNA sequences from wavelet analysis; Phys. Rev. Lett. 74 3293-3296

Crick F H C 1968 The origin of the genetic code; J. Mol. Biol. 38 367-379

Davies P 1998 The fifth miracle (London: Penguin Books)

Grover L 1996 A fast quantum mechanical algorithm for data- base search; Proc. 28th Annual ACM Symposium on Theory of Computing (Philadelphia) pp 212-219 [quant-ph/9605043]

Hayes B 1998 The invention of the genetic code; Am. Sci. 86 $8-14$

Lewin B 2000 Genes VII (Oxford: Oxford University Press)

Nandy A 1996 Graphical analysis of DNA sequence structure: III. Indications of evolutionary distinctions and characteristics of introns and exons; Curr. Sci. 70 661-668

Patel A 2000a Quantum algorithms and the genetic code; Proc. Winter Institute on Foundations of Quantum Theory and Quantum Optics (Calcutta), Pramāna 56 367-381 [quant$\mathrm{ph} / 0002037]$

Patel A 2000b Quantum database search can do without sorting; Preprint IISc-CTS-1/00 [quant-ph/0012149]

Patel A 2001a Testing quantum dynamics in genetic information processing; J. Genet. 80 (in press) [quant-ph/ 0102034]

Patel A 2001b Carbon-the first frontier of information processing; Preprint IISc-CTS-8/01 [quant-ph/0103017]

Schrödinger E 1944 What is life? (Cambridge: Cambridge University Press)

von Neumann J 1958 The computer and the brain (New Haven: Yale University Press)

Watson J D, Hopkins N H, Roberts J W, Steitz J A and Weiner A M 1987 Molecular biology of the gene, 4th edition (Menlo Park: Benjamin/Cummings)

MS received 1 February 2001; accepted 2 May 2001

Corresponding editor: VIDYANAND NANJUNDIAH 